\title{
Genetics and epigenetics of myelodysplastic syndromes and response to drug therapy: new insights
}

\author{
Saeid Shahrabi, ${ }^{1}$ Abbas Khosravi, ${ }^{2}$ Mohammad Shahjahani, ${ }^{3}$ Fakher Rahim, ${ }^{2}$ \\ Najmaldin Saki ${ }^{2,3}$ \\ ${ }^{1}$ Department of Biochemistry and Hematology, Semnan University of Medical Sciences, Semnan; \\ ${ }^{2}$ Health Research Institute, Thalassemia and Hemoglobinopathy Research Center, Ahvaz \\ Jundishapur University of Medical Sciences, Ahvaz; ${ }^{3}$ Golestan Hospital Clinical Research \\ Development Unit, Ahvaz Jundishapur University of Medical Sciences, Ahvaz, Iran
}

\begin{abstract}
Myelodysplastic syndromes (MDS) are a heterogeneous group of hematologic neoplasms ocurring mostly in the elderly. The clinical outcome of MDS patients is still poor despite progress in treatment approaches. About $90 \%$ of patients harbor at least one somatic mutation. This review aimed to assess the potential of molecular abnormalities in understanding pathogenesis, prognosis, diagnosis and in guiding choice of proper therapy in MDS patients. Papers related to this topic from 2000 to 2016 in PubMed and Scopus databases were searched and studied. The most common molecular abnormalities were TET2, ASXL1 as well as molecules involved in spliceosome machinery (U2AF1, SRSF2 and SF3B1). Patients with defects in TET2 molecule show better response to treatment with azacitidine. IDH and DNMT3A mutations are associated with a good response to decitabine therapy. In addition, patients with del5q subtype harboring TP53 mutation do not show a good response to lenalidomide therapy.
\end{abstract}

Correspondence: Najmaldin Saki, Health Research Institute, Research Center of Thalassemia \& Hemoglobinopathy, Ahvaz Jundishapur University of Medical Sciences, Ahvaz, Iran. E-mail: najmaldinsaki@gmail.com

Key words: Myelodysplastic syndromes; genetic; epigenetic.

Acknowledgements: we wish to thank all our colleagues in Golestan hospital clinical research development unit, Ahvaz Jundishapur University of Medical Sciences. We wish to thank R. E. Kast, MD at The IIAIGC Study Center for helpful suggestions.

Contributions: NS conceived the manuscript and revised it; SSh, KhA, MSh and FR wrote the manuscript and prepared the Table and the Figure.

Conflict of interest: the authors declare that they have no conflict of interest.

Received for publication: 27 August 2016.

Accepted for publication: 6 December 2016.

This work is licensed under a Creative Commons Attribution NonCommercial 4.0 License (CC BY-NC 4.0).

(C) Copyright S. Shahrabi et al., 2016

Licensee PAGEPress, Italy

Oncology Reviews 2016; 10:311

doi:10.4081/oncol.2016.311
In general, the results of this study show that molecular abnormalities can be associated with the occurrence of a specific morphological phenotype in patients. Therefore, considering the morphology of patients, different gene profiling methods can be selected to choice the most appropriate therapeutic measure in these patients in addition to faster and more cost-effective diagnosis of molecular abnormalities.

\section{Introduction}

Myelodysplastic syndromes (MDS) form a heterogeneous group of bone marrow (BM) disorders that often occur in the elderly and in those who have had a history of contact with cytotoxic agents. ${ }^{1}$ These patients generally have varying cytopenia in different lineages due to ineffective hematopoiesis. Morphological dysplasia in BM as well as acute myelogenous leukemia (AML) transformation occurs in a third of patients. ${ }^{2}$ Diagnosis of MDS is still based on the assessment of peripheral blood smear, blood cell count, BM aspiration and biopsy to evaluate the cellularity and fibrosis. ${ }^{3}$

Treatment of MDS patients is different based on their prognosis score, ranging from simple supportive care such as blood transfusion to advanced treatments like BM transplantation. Currently, three chemotherapeutic drugs have been approved by FDA, including azacitidine and decitabine as the first-line therapy for all subgroups of MDS as well as Lenalidomide for MDS with $5 q$ deletion, which can improve hematopoiesis, delay disease progression, increase survival and improve quality of life in a number of patients. ${ }^{4}$ It should be noted that allogeneic hematopoietic stem cell transplantation is the only potentially curative therapy for MDS patients, but it is useful only for a limited number of patients given the usually advanced age of patients. ${ }^{5}$ Different prognosis systems have also been suggested for MDS patients that classify patients based on the number of cytopenic lineages, percentage of BM blasts and cytogenetic abnormalities. ${ }^{6}$ Pathophysiology of MDS is a manifold process in which cytogenetic changes, gene mutations (or both) as well as extensive gene hypermethylation in advanced stages of the disease are involved. ${ }^{7}$ Somatic mutations commonly arise in MDS patients and are associated with a number of clinical features in patients, including p53 and NRAS mutations that are strongly correlated with severe thrombocytopenia. ${ }^{8}$ It has also been shown that different genes are mutated during aging, which are generally associated with transformation to myeloid/lymphoid leukemia, including TET2, DNMT3A, ASXL1 and SF3B1. ${ }^{9}$ This may be a reason for the incidence of MDS in seventh and eighth decades of life. ${ }^{10}$ However, the prognosis system of these mutations has not been taken into account and since these mutations generally occur in genes 
encoding spliceosome components, chromatin remodeling genes, epigenetic pattern regulators as well as transcription factors, their use in target therapy is faced with problems. ${ }^{11}$

It seems that progress in treatment of MDS patients is dependent upon accurate identification of disease mechanisms; therefore, attempts to improve current therapeutic methods should be based on understanding specific disease-causing mechanisms. There are several obstacles in the use of gene mutations as therapeutic targets as well as diagnostic markers of disease. ${ }^{12}$ Gene mutations are involved in MDS pathogenesis and have a high potential for guiding proper choice of therapeutic approach for patients. This study thus aimed to identify the role of gene mutations involved in MDS pathogenesis as well as ability of specific gene mutations topredict response to different drugs in case of multiple mutations.

\section{Genetic and epigenetic abnormalities}

Development of next generation sequencing (NGS) techniques led to detection of somatic gene mutations in approximately $90 \%$ of MDS patients. The currently detected recurrent mutations involve nearly 30 genes, which are mainly classified in four functional groups ${ }^{13}$ (Table 1) listed 1.

\section{Oncogenes and tumor suppressors}

TP53: p53 protein is a transcription factor expressed by TP53 gene in 17 p13.1 locus. This protein acts as a tumor suppressor regulating gene expression in response to stress signals of the cell, which leads to induction of cell cycle arrest, apoptosis, aging, DNA repair or changes in cellular metabolism. ${ }^{14}$ TP53 mutation is common in solid tumors and has been reported as a common mutation in hematologic malignancies to a lesser extent. ${ }^{15}$ Mouse double minute 2 homolog (MDM2) bind to and inhibits function of p53. Deregulation of p53 pathway is either secondary to mutation in TP53 gene or due to changes in the expression of its inhibitor of MDM2. ${ }^{16}$ Although TP53 mutation is observed in about $10 \%$ of all MDS patients, it is seen in $20 \%$ of del $5 q$ and in over $70 \%$ of complex karyotype patients, respectively. ${ }^{13,17}$ However, increased expression of MDM2 is observed only in 10\% of MDS patients. ${ }^{17}$ The importance of p53 mutation in del5q subtype is related to RPS 14. This protein, which binds and inhibits MDM2, is subject to reduced expression in del5q. ${ }^{18}$ Therefore, when p53 mutation occurs along with del5q, p53 pathway is dysregulated in two ways. It has also been shown that this mutation is associated with thrombocytopenia and increased blasts, which is a predictor of poor prognosis in MDS patients. $^{8}$

N/K RAS: Members of RAS protein family include N-Ras, K-RAS and H-RAS. As a vital hub, Ras-GTPases are involved in several signaling pathways and play a role in regulating different cellular processes, including proliferation, differentiation, self-renewal and apoptosis. ${ }^{19}$ Activating mutations or Ras gene amplification is observed in nearly a third of human cancers. ${ }^{20}$ These mutations lead to non-ligand-dependent activation of signaling pathways, leading to increased cell proliferation. Mutations involving Ras gene mutations, most notably NRAS, are observed in 5-10\% of MDS patients; however, the prevalence of this mutation is up to $30 \%$ in patients with isolated isochromosome 17 [i (17q) ]. ${ }^{21}$ These mutations have been associated with poor prognosis in the majority (but not all) the studies. ${ }^{22}$ NRAS mutation also seems to play an important role in AML transformation of these patients, such that $26 \%$ of AML patients showed this mutation at the time of transformation. ${ }^{23}$

Ecotropic viral integration site 1 (EVI1): EVI1 gene (3q26) is expressed in a low level in normal blood and BM cells. ${ }^{24}$ EVI1 is a zinc finger protein regulating the activity of transcription factors during dif- ferent events. This protein regulates various cellular mechanisms such as proliferation, differentiation and apoptosis. EVI1 causes changes in the activity of transcription factors and signaling pathways, including GATA andRunt-related transcription factor 1 (RUNX1). ${ }^{25}$ GATA1 is an essential transcription factor in erythropoiesis and megakaryopoiesis, and EVI1 can inhibit GATA-1 dependent erythropoiesis as an antagonist. $^{26,27}$ In addition, EVI1 suppresses the PU.1 and RUNX1 transcription factors involved in myeloid differentiation through interaction with them, ultimately disrupting the expression of genes involved in terminal myeloid differentiation. ${ }^{28,29}$ EVI1 also regulates the maintenance and repopulation of hematopoietic stem cells as well as HSCs transformation into leukemic cells via interaction with GATA2 and phosphatase and tensin homolog (PTEN) transcription factors. ${ }^{30,31}$ Therefore, EVI1 generates leukemic cells in addition to suppressing the differentiation and maturation of erythrocytes, megakaryocytes and myeloid cells. Aberrant expression of EVIl has been observed in BM of AML patients and approximately $10 \%$ of MDS patients. These patients generally have a poor prognosis and show manifestations of multi-lineage dysplasia, severe anemia and general cytopenia. It is also noteworthy that increased expression of EVI1 is observed in chromosomal rearrangements involving $3 q 26 .^{29}$ Moreover, it seems that aberrant expression of EVI1 is closely related with hypermethylation of P15 (a cyclin dependent kinase inhibitor) promoter in MDS patients, which results in decreased expression of P15 and interference with the cell cycle. ${ }^{32}$ These findings can indicate one of the most important mechanisms of MDS neoplasia by EVI1. Therefore, evaluation of the effect of HMA drugs on patients with increased expression of EVI1 can present valuable results.

\section{DNA methylation}

DNA Methyltransferases (DNMTs): DNA methylation, the addition of a methyl group added to cytosine or adenine, is an epigenetic change that plays an important role in transcription regulation, chromatin remodeling and genomic instability. ${ }^{33}$ In general, DNA methylation tends to inhibit transcription. In cancers generally, tumor suppressor genes tend to be hypermethylated and oncogenes tend to be hypomethylated.

In fact, the pattern of DNA methylation determines chromatin structure, which is substantially changed in neoplasms. These epigenetic changes generally lead to promoter hypermethylation and the resulting aberrant silencing of genes, a process that can initiate tumor progression. ${ }^{34}$ DNA methyltransferases (DNMTs) are key DNA methylation enzymes, including DNMT1, DNMT3A and DNMT3B, which cause DNA methylation in carbon 5 of cysteine in CPG islands, which generates $5 \mathrm{mC}$. $5 \mathrm{mC}$ causes steady inhibition of gene expression. DNMTs, especially DNMT3A, are subject to several mutations in hematologic malignancies, ${ }^{35}$ so that mutations involving DNMT3A have been observed in $7 \%$ of MDS patients. ${ }^{36}$ The mechanism by which DNMT3A results in leukemia is not clear, but this mutation seems to be insufficient to cause leukemia and only predisposes to it, while it interestingly occurs simultaneous with SF3B1 and U2AF1 mutations. ${ }^{37}$ These findings can imply coordination between these mutations to cause MDS. This mutation has been associated with poor prognosis as patients with DNMT3A mutation have lower overall survival and a higher risk of transformation to AML relative to those lacking it. ${ }^{36}$

Ten-eleven translocation 2 (TET2): TET protein family (TET1, TET2 and TET3) includes dioxygenases related to $\mathrm{Fe} 2+$ and oxoglutarate that can oxidize $5 \mathrm{mC}$ to $5 \mathrm{hmC}, 5 \mathrm{fC}$ and $5 \mathrm{caC}$ in DNA and thus cause DNA demethylation. ${ }^{38}$ These proteins are therefore involved in different biological processes such as epigenetic regulation of gene transcription, embryonic development, stem cell function and leukemic transformation. ${ }^{39}$ It seems that each of these genes is expressed in a specific tissue in order to control a particular gene panel in that tissue. Among them, TET2 plays an important role in hematopoiesis and differentia- 
tion induction of hematopoietic cells (40). TET2 is mutated in many hematologic malignancies and is one of the most common mutations found in MDS patients, so that $19-23 \%$ of patients show this mutation. ${ }^{41-43}$ TET2 mutations lead to loss of normal activity or decreased expression of this protein, and patients with these mutations show a sharp decline in $5 \mathrm{hmC}$ as well as $5 \mathrm{mC}$ accumulation. ${ }^{44,45}$ However, it is reported that this mutation is indicative of a good prognosis in MDS patients. ${ }^{46}$

Isocitrate dehydrogenase (IDH): IDH1 (2q33.3) and its mitochondrial homolog, IDH2 (15q.26.1), which encode the enzymes active in the Krebs cycle. These enzymes cause oxidative decarboxylation of isocitrate and convert it to alpha-ketoglutarate. ${ }^{47}$ Mutations of these enzymes are of gain of function type and in their new form they trigger the production of 2-hydroxyglutarate. ${ }^{47}$ Accumulation of 2-hydroxyglu- tarate inhibits the alpha-ketoglutarate-dependent enzymes, including TET2. Therefore, IDH1/IDH2 mutations cause epigenetic defects similar to TET2 mutation, which impair myeloid differentiation and result in increased expression of stem/progenitor cell markers. ${ }^{48}$ Besides, the accumulation of 2-hydroxyglutarate leads to production of reactive oxygen species (ROS), damage to DNA, inhibition of Egg-laying defective Nine (EGLN) followed by stabilization of hypoxia-inducible factor 1a (HIF-1a). ${ }^{49,50}$ However, it has been shown that damage to DNA, especially activation of HIF-1a, plays an important role in pathogenesis of MDS. ${ }^{51,51}$ IDH mutations are seen in about $3-12 \%$ of MDS patients and are associated with poor clinical prognosis in these patients. ${ }^{53}$

\section{Histone modification}

Enhancer of zeste homolog 2 (EZH2): EZH2 is a histone methyltrans-

Table 1. Common molecular defects in myelodysplastic syndromes.

\begin{tabular}{|c|c|c|c|c|c|c|c|c|}
\hline $\begin{array}{l}\text { Oncogenes/ } \\
\text { Tumor } \\
\text { supressor }\end{array}$ & Chro. & Function & Incidence & $\begin{array}{l}\text { Related } \\
\text { chromosome }\end{array}$ & $\begin{array}{c}\text { Morphologically } \\
\text { subtype } \\
\text { aberration }\end{array}$ & $\begin{array}{l}\text { Clinical } \\
\text { features }\end{array}$ & Prognosis & Ref. \\
\hline TP53 & 17p13.1 & $\begin{array}{l}\text { Tumor supressor } \\
\text { gene }\end{array}$ & $10 \%$ & $\begin{array}{l}\text { Complex karyotype, } \\
\text { del5q }\end{array}$ & $\operatorname{del} 5 q$ & $\begin{array}{l}\text { Higher blast } \\
\text { count, blood } \\
\text { transfusion } \\
\text { dependent, } \\
\text { severe } \\
\text { thrombocytopenia }\end{array}$ & Poor & 8,14 \\
\hline NRAS & 1p13.2 & $\begin{array}{l}\text { Oncogene, } \\
\text { GTPase }\end{array}$ & $4 \%$ & $\mathrm{i}(17 \mathrm{q})$ & - & $\begin{array}{l}\text { Severe } \\
\text { thrombocytopenia }\end{array}$ & & \\
\hline EVI1 & $3 q 26$ & $\begin{array}{l}\text { Transcriptional } \\
\text { regulator and } \\
\text { oncoprotein } \\
\text { that interact }\end{array}$ & $10 \%$ & $3 q 26$ & - & $\begin{array}{l}\text { Severe anemia, } \\
\text { multilineage myeloid } \\
\text { dysplasia }\end{array}$ & Poor & 29 \\
\hline
\end{tabular}

that interact with PU.1

\begin{tabular}{|c|c|c|c|c|c|c|c|}
\hline TET2 & $4 q 24$ & $\begin{array}{l}\text { Methylcytosine } \\
\text { dioxygenase }\end{array}$ & $19-23 \%$ & Normal & $\begin{array}{l}\text { No significantly differ } \\
\text { in clinical/hematologic } \\
\text { parameters }\end{array}$ & Good & 8,46 \\
\hline IDH1/IDH2 & $\begin{array}{l}2 q 33.3 \\
\text { (IDH1) } \\
15 q .26 .1 \\
\text { (IDH2) }\end{array}$ & $\begin{array}{l}\text { Catalyze the oxidative } \\
\text { decarboxylation of } \\
\text { isocitrate to 2- } \\
\text { oxoglutarate }\end{array}$ & $3.4-12.2 \%$ & $\begin{array}{l}\text { Normal karyotype and RAEB-1/RAEB-2 } \\
-7 / 7 \mathrm{q}\end{array}$ & - & Poor & 47,53 \\
\hline DNMT3A & 2p23.3 & DNA methylteransferas & $7.8 \%$ & Normal & No impact & Poor & 36 \\
\hline EZH2 & $7 q 35-q 36$ & Histone methyltransfer & case $6 \%$ & $\operatorname{del} 7 / 7 q$ & - & Poor & 56,57 \\
\hline ASXL1 & $20 q 11.2$ & Histone-binding proteir & $20 \%$ & $\begin{array}{l}\text { Complex karyotypes } \\
\text { i(17q) }\end{array}$ & - & Poor & $21,61,70$ \\
\hline SF3B1 & $2 q 33.1$ & Splicing & $16 \%$ & -5 or $-5 q$ & Refractory anemia & Not predict & $65,69-71$ \\
\hline U2FAl & 21q22.3 & Splicing & $15 \%$ & Trisomy 8 and del (20q) & - & Not predict & $65,69-71$ \\
\hline SRSF2 & $17 q 25.1$ & Splicing & $13 \%$ & $\begin{array}{l}\text { CMML } \\
\text { RAEB }\end{array}$ & - & Poor & $65,69-71$ \\
\hline RUNX1 & 21q22 & Transcription factor & $12 \%$ & RAEB, RAEB-t & $\begin{array}{l}\text { Higher neutrophil } \\
\text { counts, severe } \\
\text { thrombocytopenia }\end{array}$ & Poor & $8,72-74$ \\
\hline JAK2 & 9p24.1 & Tyrosine kinase & $50 \%$ in RARS & RARS & - & Not studied & $21,75-76$ \\
\hline CBL & $11 q 23.3$ & $\begin{array}{l}\text { Targeting substrates } \\
\text { for degradation } \\
\text { by the proteasome }\end{array}$ & 5\% in CMML & CMML & - & Poor & 77 \\
\hline RPS14 & $5 q 33.1$ & $\begin{array}{l}\text { Ribosomal protein } \\
\text { S14; a component } \\
\text { of the 40S subunit }\end{array}$ & $100 \%$ in del $5 q$ & del5q & Anemia & Not studied & 22,79 \\
\hline
\end{tabular}

EVI1, ecotropic viral integration site 1; TET2, ten-eleven translocation 2; IDH, isocitrate dehydrogenase; RAEB, refractory anemia with excess blasts; DNMT3A, DNA methyltransferase 3A; EZH2, enhancer of zeste homolog 2; ASXL1, additional sex combs like 1; i(17q), isolated isochromosome 17; SF3B1, splicing factor 3B subunit 1; RARS-T, refractory anemia with ring sideroblasts associated with thrombocytosis; U2AF1, U2 small nuclear RNA auxiliary factor 1; CMML, chronic myelomonocytic leukemia; SRSF2, serine/arginine-rich splicing factor 2; RUNX1, runt-related transcription factor 1; RAEB-t, refractory anemia with excess blasts in transformation; JAK2, Janus kinase 2; CBL, C-cbl E3 ubiquitin ligase gene; RPS14, ribosomal protein S14. 
ferase from polycomb protein group (PcG) making the catalytic subunit of polycomb repressive complex2 (PRC2) and inhibiting gene expression via methylation of histone $\mathrm{H} 3$ lysine 27 (H3K27). ${ }^{54}$ It appears that this protein has the dual role of oncogene and tumor suppressor as both overexpression and loss of function mutations of it are associated with malignancy, especially MDS. ${ }^{55}$

EZH2 mutation has been detected in approximately $6 \%$ of MDS patients and is a predictor of poor prognosis for these patients. ${ }^{56} \mathrm{EZH} 2$ is located on $7 \mathrm{q} 36.1$ locus, whereas deletion of chromosome 7 or $7 \mathrm{q}$ as the locus of this gene is a common chromosomal abnormality in MDS patients. ${ }^{57}$ Therefore, it seems that in addition to mutations involving EZH2, chromosomal abnormalities in chromosome 7 play a role in MDS pathogenesis through the impact on EZH2. Nevertheless, it has been shown in mouse models that mere deletion of EZH2 can induce MDS/MPN like disease. ${ }^{58}$ In general, these findings suggest that EZH2 can be a key factor in pathogenesis of MDS, which has a high potential for targeted therapy.

Additional sex combs like 1 (ASXL1): ASXL1 is located on 20q11 locus and is another enzyme involved in histone methylation. ${ }^{59}$ ASXL1 causes changes in histones via interaction with PRC2 components such as EZH2. ${ }^{60}$ ASXL1 mutation is observed in about $20 \%$ of MDS patients and is indicative of poor prognosis in these patients. ${ }^{61}$ It was shown that ASXLI mutation is associated with reduced transformation time of MDS to AML, especially chronic myelomonocytic leukemia (CMML) (62). However, it has recently been reported that ASXL1 results in MDS transformation to AML through mutation in SET binding protein1 (SETBP1), which prevents its ubiquitination, although other genes like GATA2 and NRAS have been reported in this regard. ${ }^{62,63}$ Understanding the accurate role of ASXL1 pathogenesis needs further investigations.

\section{Splicesome}

It was recently shown that mutations (mainly heterozygous) in genes involved in splicing machinery such as Splicing factor 3B subunit 1 (SF3B1), Serine/Arginine-Rich Splicing Factor 2 (SRSF2) and U2 Small Nuclear RNA Auxiliary Factor 1 (U2AF1) play an important role in pathogenesis of myelodysplasia. ${ }^{64}$ SF3B1 mutation is the first known mutation in the genes involved in splicing machinery and has an unexpectedly high incidence in Refractory Anaemia with Ring Sideroblasts (RARS) (68-75\%) and Refractory anemia with ringed sideroblasts associated with marked thrombocytosis (RARS-T) (81\%) subtypes, so that it seems to play an important role in pathogenesis of the mentioned subtypes. ${ }^{65}$

SF3B1 mutation will reduce the function of several genes involved in mitochondrial function. Therefore, according to heme synthesis in mitochondria, increase in the number of ringed sideroblasts due to impaired synthesis of heme is a finding frequently observed in patients harboring SF3B1 mutation. ${ }^{66}$ However, some studies have indicated the reduced transcription and abnormal splicing of genes associated with heme synthesis, including ABCB7 in presence of SF3B1 mutation. ${ }^{67}$ Although SF3B1 mutation is mainly observed in RARS subtype associated with increasing platelet count, U2FA1 and SRSF2 mutations are mostly observed in CMML and RAEB1/2 subtypes, and up to $47 \%$ of CMML patients have been reported to harbor SRFS2 mutation. ${ }^{65,68-70}$ SRSF2 mutation is predictor of poor prognosis and accelerated transformation to AML, while the other two mutations have no effect on the outcome of patients. ${ }^{71}$

\section{Others}

RUNX1, Janus kinase 2 (JAK2) and C-cbl E3 ubiquitin ligase gene (CBL) are other genes that are commonly mutated in MDS patients. RUNX1 gene (21q22) encodes a subunit of core binding factor (CBF). This transcription factor is commonly dysregulated in hematologic malignancies. ${ }^{72}$ RUNX1 mutation is observed in $12 \%$ of MDS patients at diagnosis and is generally associated with refractory anemia with excess blasts (RAEB) and refractory anemia with excess blasts in transformation (RAEB-t) subtypes (23.6\% of cases) ${ }^{73,74}$ In addition, patients harboring this mutation show a poor prognosis. ${ }^{74}$

JAK2 V617F mutation in hematopoietic stem cells increases the sensitivity to erythropoietin and growth factor independent growth. This mutation is commonly observed in Philadelphia negative myeloproliferative disease (MPD). However, the incidence of this mutation has recently been shown also in MDS patients. ${ }^{75}$ Although the incidence of JAK2 V617F mutation is rare in MDS, it is significantly increased in RARS patients, especially RARS with excessive platelets and/or obvious BM fibrosis, showing an approximate $50 \%$ incidence in this subgroup. ${ }^{76}$ This mutation is also associated with isolated isochromosome $17 \mathrm{q} \cdot{ }^{21}$ In addition, CBL which encodes an enzyme involved in the degradation of tyrosine kinase receptor, is mutated in MDS patients. JAK2 V617F increases growth signals and proliferation and is associated with poor prognosis. ${ }^{77}$

\section{MDS with del5q}

del5q is a better known subtype of MDS observed in 5-10\% of cases, which shows severe anemia, neutropenia and normal or increased platelet count with good prognosis. ${ }^{78}$ Commonly deleted region (CDR) in $5 \mathrm{q}$ - includes a region of approximately $1.5 \mathrm{Mb}$ that decreases the expression of genes in this region (probably due to haploinsufficiency). These genes include those encoding the Ribosomal Protein S14 (RPS14), SPARC and serine-threonine kinase CSNK1A1 in 7\% of cases.22 Decreased expression of RPS14 was shown to play a vital role in development of anemia (a feature of this subtype), and the induction of its expression can reduce disease symptoms. ${ }^{79}$ Allelic exclusion of RPS14 gene disrupts the integrity of mitochondrial membrane, which subsequently leads to the release of ribosomal proteins and nuclear stress, which would result in MDM2 uncoupling and p53 activation. ${ }^{80}$ Furthermore, miR145 and miR146 are two important miRNA molecules located in 5q region and highly expressed in HSCs. MiR 145 directly targets MAL/TRAP proteins and FLil proteins whereas TRAF6 protein is the main target of miR $146 .{ }^{81}$ Knockout of these miR molecules showed that although none of them play a role in anemia, miR146 reduction causes neutropenia through increased TRAF6.Reduction of miR145 causes thrombosis via increasing MAL and FLI levels. ${ }^{82}$ These findings suggest that haploinsufficiency of RSP14, miR146 and miR145 are responsible for anemia, neutropenia and thrombocytosis in patients with del $5 q$, respectively.

\section{MDS response to drug therapies}

Although there have been improvements in treatment of MDS patients, the clinical outcome of patients is still poor. Some studies have provided evidence indicating that the existence of some mutations is associated with response to specific treatments. As mentioned, 5-azcitidine, decitabine, deferasirox and lenlidomide have received FDA approval for treatment of MDS. ${ }^{83}$ In relation to $\mathrm{p} 53$ mutation, it has been shown that response to treatment with azacitidine is not associated with p53 mutation. ${ }^{16}$ However, it appears that patients with del $5 q$ harboring this mutation respond poorly to therapy with lenalidomide. ${ }^{84}$

This may be related to therapeutic mechanism of lenalidomide. CSNK1A1 gene on CDR of $5 q$ chromosome encodes casein kinase 1 a (CK1a), a tyrosine kinase with a variety of cellular functions. Inhibition of p53 via stabilization of MDM2 and MDMX is one of the most important functions of CKla. Lenalidomide decreases the level of CKla pro- 
tein; therefore, due to haploinsufficiency of del5q cells for CSNK1A1 gene, these cells are more sensitive to lenalidomide and undergo apoptosis. ${ }^{85}$ According to these evidences, the therapeutic effect of lenalidomide is expected to decrease in presence of TP53 mutation.

In a study conducted by Bejaret al, it was found that the presence of TET2 mutation is associated with increased response to hypomethylating drugs (HMAs), especially azacitidine, when the ASXL1 gene is not mutated. ${ }^{86}$ Other studies have also partially confirmed this finding. ${ }^{87}$

The impact of mutation in the genes involved in splicing process, including SRSF2, U2AF1 and ZRSR2 on response to decitabine was studied by Hong et al. At the end of these trials, no effect on survival and response to this drug was found. ${ }^{88}$ However, in a large-scale metaanalysis study, it was shown that patients bearing IDH mutation show better response to decitabine relative to other therapies. ${ }^{53}$ Therefore, decitabine can be an appropriate choice for patients harboring IDH mutation.

The response to HMAs has been associatedwith some mutations in hematological malignancy. For example, it was reported that AML patients harboring DNMT3A mutation respond well to treatment with decitabine. ${ }^{89}$ Correlation between RAS mutations and response to treatment of MDS patients was also evaluated in a large study. The results showed that the group harboring mutant and wild type RAS had $43 \%$ and $42 \%$ response to treatment, respectively. These findings indicate that RAS mutations cause no change in response to existing treatments for MDS. ${ }^{90}$

Although these data seem to be insufficient for use in clinical practice, further studies in this field can lead to development of a predictive flow chart to choose the best therapeutic approach in patients with different mutations.

\section{In silico and computer based modeling for treatment and response prediction}

The application of mutations in detection of malignancies is possible only when correlation between them as well as their relationship with different treatments are studied given the complexity of mutations occurring in malignancies. Different bioinformatics methods have been presented that can explore these relationships in a virtual environment and then test different assumptions. In silico modeling is one of the most efficient methods in this regard.

Biophysical and biochemical principles are based on general chemistry and physics laws as well as biological processes and phenomena. Basically, the entire life is a function of chemical and physical interactions between molecules that are not alive but are specific and have relatively complex structures. Advances in computer science and technology have led to development of the idea of identification and construction of these biological processes in virtual environment. Modeling of critical systems is currently a fervent topic of bioinformatics field. This leads to addition of the term in silico to dictionary of biological sciences. For example, translation of RNA to protein is a straightforward type of simulation in biological processes.

Intra-computer simulation or in silico modeling approaches involve the process of simulating the behavior of living cells and systems. ${ }^{91,92}$ This method is a first step in structure based and rational drug design and is sometimes referred to as computer-aided drug design. Through in silico techniques, a specific drug is evaluated prior to design by various model building patterns such as QSAR or density functional theory. Similarly, in silico modeling approaches are used to develop cellular and genetic models. ${ }^{93,94}$ The integration of computer-based modeling approaches into routine techniques can improve detection of cancer and result in the so-called computer-aided diagnostic models. These approaches have been dramatically used in various cancer predictions even in hematologic malignancies such as myeloma. ${ }^{95,96}$ Information flow between in vitro, in vivo, and in silico approaches is receiving universal appreciation among scientists; thus, their role in cancer research has been highly considered in the last decades (Figure 1).

\section{Discussion and future prospective}

In this study, we investigate the genetic mutations common in MDS

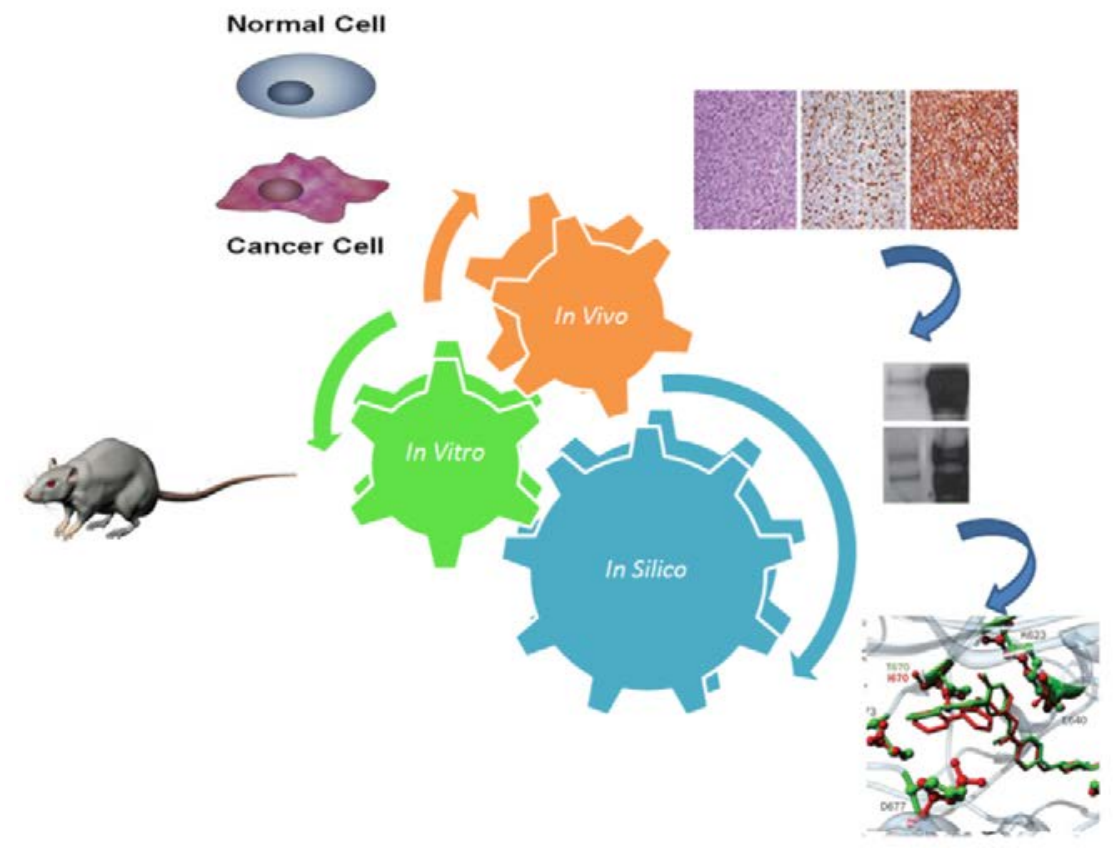

Figure 1. Schematic representation of information flow between in vitro, in vivo, and in silico approaches. The arrows illustrate data input and the feedback data used for model validation in support of further optimization. 
patients and evaluate their possible role in pathogenesis, prognosis and response to treatment in MDS patients.

The incidence of gene mutations seems to be loosely associated with phenotypic subtype of disease, specific chromosomal abnormalities as well as other mutations.

Mutation in SF3B1 and JAK2 is strongly correlated with the incidence of RARS and RARS-T phenotypes. ${ }^{61,65,76}$ Moreover, RUNX1 mutation rate has been reported to be associated with RAEB and RAEB-T and mutation in SRSF2 and U2AF1 is associated with CMML. ${ }^{65,73,74}$ This coincidence of different mutations has been reported in some cases. DNMT3A mutation is associated with SF3B1 and U2AF1 and is significantly associated with SRSF2 and ASXL1. ${ }^{37}$ Furthermore, the incidence of IDH mutations is shown to be closely related with ASXL1, SRSF2 and DNMT3A mutations. ${ }^{74}$ EZH2 mutations are also associated with the incidence of RUNX1 in MDS patients. ${ }^{97}$ In addition, SRSF2 and ZRSR2 mutations in patients harboring TET2 mutation as well as U2AF1 mutation are associated with abnormality in chromosome 20 and ASXL1 gene mutation. ${ }^{78}$

Therefore, further understanding of concurrent genetic mutations as well as their relationship with phenotype of each patient can lead to assessment of different genetic profiles of patients. This will accelerate the detection of molecular abnormalities and reduce the costs of final diagnosis of patients. Given the different responses to treatments with the incidence of gene mutations, the best therapeutic approach for these patients can be ultimately specified. In general, this approach eventually leads to development of an individual-based system of treatment. However, given the above-mentioned complexities, this can only be achieved through bioinformatics modeling systems. It is suggested that in future studies, while searching for further information on the relationship between molecular abnormalities with disease phenotype and response to different drugs, concurrence of mutations with each other is studied to develop computer models to interpret such data.

\section{References}

1. Garcia Manero G. Myelodysplastic syndromes: 2015 Update on diagnosis, risk stratification and management. Am J Hematol 2015;90:831-41.

2. Troy JD, Atallah E, Geyer JT, Saber W. Myelodysplastic syndromes in the United States: an update for clinicians. Ann Med 2014;46:283-9.

3. Malcovati L, Hellström-Lindberg E, Bowen D, et al. Diagnosis and treatment of primary myelodysplastic syndromes in adults: recommendations from the European LeukemiaNet. Blood 2013;122:2943-64.

4. Saunthararajah Y. Key clinical observations after 5-azacytidine and decitabine treatment of myelodysplastic syndromes suggest practical solutions for better outcomes. ASH Education Program Book 2013;2013:511-21.

5. Tefferi A, Vardiman JW. Myelodysplastic syndromes. N Engl J Med 2009;361:1872-85.

6. Haase D, Germing U, Schanz J, et al. New insights into the prognostic impact of the karyotype in MDS and correlation with subtypes: evidence from a core dataset of 2124 patients. Blood 2007;110:4385-95.

7. Adès L, Itzykson R, Fenaux P. Myelodysplastic syndromes. Lancet 2014;383:2239-52.

8. Bejar R, Stevenson K, Abdel-Wahab 0, et al. Clinical effect of point mutations in myelodysplastic syndromes. $N$ Engl $\mathrm{J}$ Med 2011;364:2496-506.

9. Xie M, Lu C, Wang J, et al. Age-related mutations associated with clonal hematopoietic expansion and malignancies. Nature Med
2014;20:1472-8.

10. Cogle CR. Incidence and burden of the myelodysplastic syndromes. Curr Hematol Malign Rep 2015;10:272-81.

11. Steensma DP, ed. Myelodysplastic syndromes: diagnosis and treatment. Mayo Clinic Proceedings. Amsterdam: Elsevier; 2015.

12. Bejar R. Myelodysplastic syndromes diagnosis: what is the role of molecular testing? Curr Hematol Malign Rep 2015;10:282-91.

13. Itzykson R, Kosmider 0, Fenaux P. Somatic mutations and epigenetic abnormalities in myelodysplastic syndromes. Best Pract Res Clin Haematol 2013;26:355-64.

14. Khosravi A, Shahrabi S, Shahjahani M, Saki N. The bone marrow metastasis niche in retinoblastoma. Cell Oncol 2015;38:253-63.

15. Olivier M, Hollstein M, Hainaut P. TP53 mutations in human cancers: origins, consequences, and clinical use. Cold Spring Harbor Perspect Biol 2010;2:a001008.

16. Kulasekararaj AG, Smith AE, Mian SA, et al. TP53 mutations in myelodysplastic syndrome are strongly correlated with aberrations of chromosome 5 , and correlate with adverse prognosis. Br J Haematol 2013;160:660-72.

17. McGraw KL, Cluzeau T, Sallman DA, et al. TP53 and MDM2 single nucleotide polymorphisms influence survival in non-del $(5 q)$ myelodysplastic syndromes. Oncotarget 2015;6:34437-45.

18. Zhou X, Hao Q, Liao J, et al. Ribosomal protein S14 unties the MDM2-p53 loop upon ribosomal stress. Oncogene 2013;32:388-96.

19. Niemeyer CM. RAS diseases in children. Haematologica 2014;99:1653-62.

20. Bertacchini J, Ketabchi N, Mediani L, et al. Inhibition of Rasmediated signaling pathways in CML stem cells. Cell Oncol 2015:1-12.

21. Kanagal-Shamanna R, Luthra R, Yin CC, et al. Myeloid neoplasms with isolated isochromosome $17 \mathrm{q}$ demonstrate a high frequency of mutations in SETBP1, SRSF2, ASXL1 and NRAS. Oncotarget 2016 [Epub ahead of print].

22. Pellagatti A, Boultwood J. The molecular pathogenesis of the myelodysplastic syndromes. Eur J Haematol 2015 [Epub ahead of print].

23. Badar T, Patel KP, Thompson PA, et al. Detectable FLT3-ITD or RAS mutation at the time of transformation from MDS to AML predicts for very poor outcomes. Leuk Res 2015;39:1367-74.

24. Glass C, Wilson M, Gonzalez R, et al. The role of EVI1 in myeloid malignancies. Blood Cell Mol Dis 2014;53:67-76.

25. Smol T, Nibourel 0, Marceau-Renaut A, et al. Quantification of EVI1 transcript levels in acute myeloid leukemia by RT-qPCR analysis: a study by the ALFA Group. Leuk Res 2015 [Epub ahead of print].

26. Vinatzer U, Taplick J, Seiser C, et al. The leukaemia-associated transcription factors EVI-1 and MDS1/EVI1 repress transcription and interact with histone deacetylase. $\mathrm{Br} \mathrm{J}$ Haematol 2001;114:566-73.

27. Welch JJ, Watts JA, Vakoc CR, et al. Global regulation of erythroid gene expression by transcription factor GATA-1. Blood 2004;104:3136-47.

28. Senyuk V, Sinha KK, Li D, et al. Repression of RUNX1 activity by EVI1: a new role of EVI1 in leukemogenesis. Cancer Res 2007;67:5658-66.

29. Laricchia-Robbio L, Premanand K, Rinaldi CR, Nucifora G. EVI1 Impairs myelopoiesis by deregulation of PU. 1 function. Cancer Res 2009;69:1633-42.

30. Goyama S, Yamamoto G, Shimabe M, et al. Evi-1 is a critical regulator for hematopoietic stem cells and transformed leukemic cells. Cell Stem Cell 2008;3:207-20.

31. Yamazaki H, Suzuki M, Otsuki A, et al. A remote GATA2 hematopoietic enhancer drives leukemogenesis in inv (3)(q21; q26) by activating EVI1 expression. Cancer Cell 2014;25:415-27. 
32. Ohashi H, Tsushita K, Utsumi M, et al. Relationship between methylation of the p15 gene and ectopic expression of the EVI-1 gene in myelodysplastic syndromes (MDS). Leukemia 2001;15:990.

33. Jones PA, Baylin SB. The fundamental role of epigenetic events in cancer. Nature Rev Genet 2002;3:415-28.

34. Taberlay PC, Jones PA. DNA methylation and cancer. Epigenet Dis 2011:1-23.

35. Li KK, Luo L-F, Shen Y, et al. DNA methyltransferases in hematologic malignancies. Semin Hematology 2013 [Epub ahead of print].

36. Lin J, Yao D-M, Qian J, et al. Recurrent DNMT3A R882 mutations in Chinese patients with acute myeloid leukemia and myelodysplastic syndrome. PLoS One 2011;6:e26906.

37. Yang L, Rau R, Goodell MA. DNMT3A in haematological malignancies. Nature Rev Cancer 2015 [Epub ahead of print].

38. Hu L, Lu J, Cheng J, et al. Structural insight into substrate preference for TET-mediated oxidation. Nature 2015;527:118-22.

39. Pastor WA, Aravind L, Rao A. TETonic shift: biological roles of TET proteins in DNA demethylation and transcription. Nature reviews. Mol Cell Biol 2013;14:341-56.

40. Branco MR, Ficz G, Reik W. Uncovering the role of 5-hydroxymethylcytosine in the epigenome. Nature Rev Genet 2012;13:713.

41. Delhommeau F, Dupont S, Valle VD, et al. Mutation in TET2 in myeloid cancers. N Engl J Med 2009;360:2289-301.

42. Smith AE, Mohamedali AM, Kulasekararaj A, et al. Next-generation sequencing of the TET2 gene in 355 MDS and CMML patients reveals low-abundance mutant clones with early origins, but indicates no definite prognostic value. Blood 2010;116:3923-32.

43. Wang J, Ai X, Gale RP, et al. TET2, ASXL1 and EZH2 mutations in Chinese with myelodysplastic syndromes. Leuk Res 2013;37:30511.

44. Ko M, An J, Pastor WA, et al. TET proteins and 5 methylcytosine oxidation in hematological cancers. Immunol Rev 2015;263:6-21.

45. Scopim-Ribeiro R, Machado-Neto JA, de Melo Campos P, et al. Low Ten-eleven-translocation 2 (TET2) transcript level is independent of TET2 mutation in patients with myeloid neoplasms. Diagn Pathol 2016;11:1.

46. Kosmider 0, Gelsi-Boyer V, Cheok M, et al. TET2 mutation is an independent favorable prognostic factor in myelodysplastic syndromes (MDSs). Blood 2009;114:3285-91.

47. Prensner JR, Chinnaiyan AM. Metabolism unhinged: IDH mutations in cancer. Nature Med 2011;17:291.

48. Im A, Sehgal A, Carroll M, et al. DNMT3A and IDH mutations in acute myeloid leukemia and other myeloid malignancies: associations with prognosis and potential treatment strategies. Leukemia 2014;28:1774-83.

49. Cohen AL, Holmen SL, Colman H. IDH1 and IDH2 mutations in gliomas. Curr Neurol Neurosci Rep 2013;13:1-7.

50. Ye D, Xiong Y, Guan K-L. The mechanisms of IDH mutations in tumorigenesis. Cell Res 2012;22:1102-4.

51. Hayashi Y, Zhang Y, Yan X, et al. HIF-1a pathway, as a signal funnel for genetic, epigenetic, and metabolic aberrations, is sufficient and essential for MDS development. Blood 2015;126:303.

52. Hole PS, Darley RL, Tonks A. Do reactive oxygen species play a role in myeloid leukemias? Blood 2011;117:5816-26.

53. Jin J, Hu C, Yu M, et al. Prognostic value of isocitrate dehydrogenase mutations in myelodysplastic syndromes: a retrospective cohort study and meta-analysis. PLoS One 2014;9:e100206.

54. McCabe MT, Ott HM, Ganji G, et al. EZH2 inhibition as a therapeutic strategy for lymphoma with EZH2-activating mutations. Nature 2012;492:108-12.

55. Lund K, Adams P, Copland M. EZH2 in normal and malignant hematopoiesis. Leukemia 2014;28:44-9.

56. Xu F, Liu L, Chang CK, et al. Genomic loss of EZH2 leads to epigenetic modifications and overexpression of the HOX gene clusters in myelodysplastic syndrome. Oncotarget 2016 [Epub ahead of print].

57. Nikoloski G, Langemeijer SM, Kuiper RP, et al. Somatic mutations of the histone methyltransferase gene EZH2 in myelodysplastic syndromes. Nature Genet 2010;42:665-7.

58. Muto T, Sashida G, Oshima M, et al. Concurrent loss of Ezh2 and Tet2 cooperates in the pathogenesis of myelodysplastic disorders. J Exper Med 2013;210:2627-39.

59. Paschka P, Schlenk RF, Gaidzik VI, et al. ASXL1 mutations in younger adult patients with acute myeloid leukemia: A study of the German-Austrian Acute Myeloid Leukemia Study Group. Haematologica 2015 [Epub ahead of print].

60. Gelsi-Boyer V, Brecqueville M, Devillier R, et al. Mutations in ASXL1 are associated with poor prognosis across the spectrum of malignant myeloid diseases. J Hematol Oncol 2012;5:12.

61. Thol F, Friesen I, Damm F, et al. Prognostic significance of ASXL1 mutations in patients with myelodysplastic syndromes. J Clin Oncol 2011 [Epub ahead of print].

62. West RR, Hsu AP, Holland SM, et al. Acquired ASXL1 mutations are common in patients with inherited GATA2 mutations and correlate with myeloid transformation. Haematologica 2013 [Epub ahead of print].

63. Inoue D, Kitaura J, Matsui $\mathrm{H}$, et al. SETBP1 mutations drive leukemic transformation in ASXL1-mutated MDS. Leukemia 2015;29:847-57.

64. Yoshida K, Sanada M, Shiraishi Y, et al. Frequent pathway mutations of splicing machinery in myelodysplasia. Nature 2011;478:64-9.

65. Visconte V, Makishima H, Maciejewski J, Tiu R. Emerging roles of the spliceosomal machinery in myelodysplastic syndromes and other hematological disorders. Leukemia 2012;26:2447-54.

66. Papaemmanuil E, Cazzola M, Boultwood J, et al. Somatic SF3B1 mutation in myelodysplasia with ring sideroblasts. N Engl J Med 2011;365:1384-95.

67. Cazzola M, Rossi M, Malcovati L. Biologic and clinical significance of somatic mutations of SF3B1 in myeloid and lymphoid neoplasms. Blood 2013;121:260-9.

68. Damm F, Kosmider 0, Gelsi-Boyer V, et al. Mutations affecting mRNA splicing define distinct clinical phenotypes and correlate with patient outcome in myelodysplastic syndromes. Blood 2012;119:3211-8.

69. Makishima H, Visconte V, Sakaguchi H, et al. Mutations in the spliceosome machinery, a novel and ubiquitous pathway in leukemogenesis. Blood 2012;119:3203-10.

70. Wu L, Song L, Xu L, et al. Genetic landscape of recurrent ASXL1, U2AF1, SF3B1, SRSF2, and EZH2 mutations in 304 Chinese patients with myelodysplastic syndromes. Tumor Biol 2015:1-8.

71. Thol F, Kade S, Schlarmann C, et al. Frequency and prognostic impact of mutations in SRSF2, U2AF1, and ZRSR2 in patients with myelodysplastic syndromes. Blood 2012;119:3578-84.

72. Rio-Machín A, Menezes J, Maiques-Diaz A, et al. Abrogation of RUNX1 gene expression in de novo myelodysplastic syndrome with $\mathrm{t}(4 ; 21)(\mathrm{q} 21 ; \mathrm{q} 22)$. Haematologica 2011 [Epub ahead of print].

73. Harada $\mathrm{H}$, Harada $\mathrm{Y}$, Niimi $\mathrm{H}$, et al. High incidence of somatic mutations in the AML1/RUNX1 gene in myelodysplastic syndrome and low blast percentage myeloid leukemia with myelodysplasia. Blood 2004;103:2316-24.

74. Chen CY, Lin LI, Tang JL, et al. RUNX1 gene mutation in primary myelodysplastic syndrome-the mutation can be detected early at diagnosis or acquired during disease progression and is associat- 
ed with poor outcome. Br J Haematol 2007;139:405-14.

75. Jekarl DW, Han SB, Kim M, et al. JAK2 V617F mutation in myelodysplastic syndrome, myelodysplastic syndrome/myeloproliferative neoplasm, unclassifiable, refractory anemia with ring sideroblasts with thrombocytosis, and acute myeloid leukemia. Korean J Hematol 2010;45:46-50.

76. Hellström-Lindberg E. Significance of JAK2 and TET2 mutations in myelodysplastic syndromes. Blood Rev 2010;24:83-90.

77. Makishima H, Cazzolli H, Szpurka H, et al. Mutations of e3 ubiquitin ligase cbl family members constitute a novel common pathogenic lesion in myeloid malignancies. J Clin Oncol 2009;27:610916.

78. Raza A, Galili N. The genetic basis of phenotypic heterogeneity in myelodysplastic syndromes. Nature Rev Cancer 2012;12:849-59.

79. Ebert BL, Pretz J, Bosco J, et al. Identification of RPS14 as a 5qsyndrome gene by RNA interference screen. Nature 2008;451:3359 .

80. Wei S, Chen X, McGraw K, et al. Lenalidomide promotes p53 degradation by inhibiting MDM2 auto-ubiquitination in myelodysplastic syndrome with chromosome $5 \mathrm{q}$ deletion. Oncogene 2013;32:1110-20.

81. Starczynowski DT, Kuchenbauer F, Argiropoulos B, et al. Identification of miR-145 and miR-146a as mediators of the $5 \mathrm{q}$ syndrome phenotype. Nature Med 2010;16:49-58.

82. Rhyasen G, Starczynowski D. Deregulation of microRNAs in myelodysplastic syndrome. Leukemia 2012;2:13-22.

83. Stone RM. How I treat patients with myelodysplastic syndromes. Blood 2009;113:6296-303.

84. Jädersten M, Saft L, Smith A, et al. TP53 mutations in low-risk myelodysplastic syndromes with del $(5 q)$ predict disease progression. J Clin Oncol 2011 [Epub ahead of print].

85. Krönke J, Fink EC, Hollenbach PW, et al. Lenalidomide induces ubiquitination and degradation of CK1 [agr] in del (5q) MDS. Nature 2015 [Epub ahead of print].

86. Bejar R, Lord A, Stevenson K, et al. TET2 mutations predict response to hypomethylating agents in myelodysplastic syndrome patients. Blood 2014;124:2705-12.

87. Itzykson R, Kosmider 0, Cluzeau T, et al. Impact of TET2 muta- tions on response rate to azacitidine in myelodysplastic syndromes and low blast count acute myeloid leukemias. Leukemia 2011;25:1147-52.

88. Hong JY, Seo J-Y, Kim S-H, et al. Mutations in the spliceosomal machinery genes SRSF2, U2AF1, and ZRSR2 and response to decitabine in myelodysplastic syndrome. Anticancer Res 2015;35:3081-9.

89. Marcucci G, Metzeler KH, Schwind S, et al. Age-related prognostic impact of different types of DNMT3A mutations in adults with primary cytogenetically normal acute myeloid leukemia. J Clin Oncol 2012;30:742-50.

90. Al Kali A, Quintás Cardama A, Luthra R, et al. Prognostic impact of RAS mutations in patients with myelodysplastic syndrome. Am J Hematol 2013;88:365-9.

91. Khademvatan S, Adibpour N, Eskandari A, et al. In silico and in vitro comparative activity of novel experimental derivatives against Leishmania major and Leishmania infantum promastigotes. Exper Parasitol 2013;135:208-16.

92. Yin L, Zheng L, Xu L, et al. In-silico prediction of drug targets, biological activities, signal pathways and regulating networks of dioscin based on bioinformatics. BMC Compl Altern Med 2015;15:1.

93. Rahim F. In silico comparison of simple sequence repeats in high nucleotides-rich genomes of microorganism. Pak J Biol Sci 2008;11:2372-781.

94. Masbi MH, Mohammadiasl J, Galehdari H, et al. Characterization of wild-type and mutated RET proto-oncogene associated with familial medullary thyroid cancer. Asian Pac J Cancer Prev 2014;15:2027-33.

95. Abroun S, Saki N, Fakher R, Asghari F. Biology and bioinformatics of myeloma cell. Lab Hematol 2012;18:30-41.

96. Ghaedi H, Bastami M, Zare-Abdollahi D, et al. Bioinformatics prioritization of SNPs perturbing microRNA regulation of hematological malignancy-implicated genes. Genomics 2015;106:360-6.

97. Sashida G, Harada H, Matsui H, et al. Ezh2 loss promotes development of myelodysplastic syndrome but attenuates its predisposition to leukaemic transformation. Nature Commun 2014;5. 